\title{
Refractory B Acute Lymphoblastic Leukemia
}

National Cancer Institute

\section{Source}

National Cancer Institute. Refractory B Acute Lymphoblastic Leukemia. NCI Thesaurus.

Code C142812.

B acute lymphoblastic leukemia that does not respond to treatment. 